Gynäkologe 2013 • 46:707-708

DOI 10.1007/s00129-013-3158-2

Online publiziert: 8. Oktober 2013

(C) Springer-Verlag Berlin Heidelberg 2013

\title{
P. Husslein
}

Universitätsklinik für Frauenheilkunde, Wien, Österreich

\section{Sectio}

ein natürlicher Prozess, daher muss über deren Basisrisiko nicht aufgeklärt werden, obwohl dieses genau die Kriterien erfüllt, die eine Aufklärung bei jedem anderen medizinischen Eingriff notwendig machen würden. Aufgeklärt werden muss nämlich dann - unabhängig von der Häufigkeit des Auftretens - wenn die Komplikation „eingriffsspezifisch, schwerwiegend und von der Betroffenen nicht erwartet wird“ - die geradezu perfekte Charakterisierung beispielsweise einer Uterusruptur oder einer intrauterin erworbenen fetalen Hypoxie.

\section{1) Das Gespräch über den einzuschlagenden Geburtsweg muss ergebnisoffen geführt werden}

Die Rechtsprechung wird hier nachziehen müssen; in Zukunft wird auch rechtlich gefordert sein, was heute schon der respektvolle Umgang mit den sich uns anvertrauenden Schwangeren verlangt, nämlich ein ergebnisoffenes Gespräch über den einzuschlagenden Geburtsweg. Da$\mathrm{zu}$ sind - im Sinne einer verantwortungsvollen Aufklärung - Informationen über alle Komplikationen beider Optionen zu übermitteln, ohne Zeitdruck, möglicherweise in mehreren Gesprächen.

Es war mir wichtig in dieses Spezialheft Sectio sowohl medizinische als auch gesellschaftspolitische Aspekte einfließen $\mathrm{zu}$ lassen, aber auch die Kurz- und Langzeitkomplikationen ausführlich darzulegen.

Nimmt man die Patientenautonomie wirklich ernst, wankt der Begriff der medizinischen Indikation - jedenfalls in der Art, wie er bisher definiert ist [1]: Nicht mehr wir entscheiden, was für unsere $\mathrm{Pa}$ tienten richtig ist, sondern der Entschei- dungsprozess fällt gemeinsam - nach einem Gespräch, in das wir unsere medizinische Kompetenz und der Patient die Kompetenz über seine Lebensführung einfließen lässt - „shared decision making“, um einen ausnahmsweise wirklich zutreffenden sog. neudeutschen Begriff zu strapazieren.

Bei dieser Diskussion kann das Argument einer höheren Mortalität oder Morbidität (egal ob die Behauptung, dass diese erhöht sind, zutreffend ist oder nicht) keine so zentrale Rolle spielen, wie manche glauben - ist doch in anderen Aspekten unseres Lebens die Mortalität ebenfalls nicht das einzige Entscheidungskriterium. Sonst müsste das Bahnnetz noch viel stärker ausgebaut werden, man würde kaum mehr das Auto benutzen, und Motorrad fahren müsste überhaupt streng verboten werden. Schließlich ist das Mortalitätsrisiko bei diversen Fortbewegungsmitteln wirklich eindrucksvoll unterschiedlich: So sterben bei deren Nutzung pro Milliarde gefahrener Kilometer (http://www.asklubo.com/ de/auto-motor):

- Bahn: 0,2 Menschen

- Flugzeug: 0,4 Menschen

- Schiff: 0,5 Menschen

- Auto: 6,o Menschen

- Fahrrad: 30 Menschen

- Fußgänger: 38 Menschen

- Motorradfahrer: 45 Menschen

Die Geburtshilfe hat sich gewandelt, die zentrale Frage ist nicht mehr, wie die Kinder auf die Welt kommen. Unser Fach sollte sich mehr der Schwangerschaftsbetreuung zuwenden, dort liegen derzeit die großen Herausforderungen. Natürlich müssen wir weiter daran arbeiten, die Sectio noch ungefährlicher und schonender durchzuführen; auch müssen die Frauen besser ausgewählt werden, denen
Das wird zwar in der Rechtsprechung noch nicht so gesehen - die Geburt sei 
der Versuch der vaginalen Geburt auch tatsächlich glücken kann, bzw. diejenigen, bei denen die vaginale Geburt zu einem dauerhaften Beckenbodenschaden führen würde. Denn nicht unerwartet sind die einfache vaginale Geburt und der geplante Kaiserschnitt in den Augen der Mütter (und auch der Väter) die beliebtesten Geburtsformen, wie wir in einer aufwendigen Befragungsstudie nachweisen konnten [3]. Und das Neugeborene scheint bei einer elektiven Sectio weniger gestresst zu sein als bei einer vaginalen Geburt [4]. Ob das nun gut oder schlecht ist, darüber lässt sich trefflich streiten....

Ich hoffe, bei der Zusammenstellung der Beiträge dieses Hefts die Basis für eine stimulierende Diskussion bereitet zu haben.

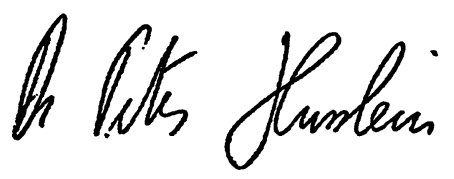

Univ.-Prof. Dr. Peter Husslein

\section{Korrespondenzadresse}

Univ.-Prof. Dr. P. Husslein

Universitätsklinik für Frauenheilkunde Währinger-Gürtel 18-20, 1090 Wien

Österreich

peter.husslein@meduniwien.ac.at

\section{Literatur}

1. Husslein $P$ (2010) Patientenautonomie - und was sich daraus ergibt, wenn man sie tatsächlich ernst nimmt... Speculum 28(4):5-6

2. Husslein P, Metka M (1997) Lassen Sie sich heute doch einmal provozieren. Speculum 15(3):3-6

3. Schindl M, Birner P, Reingrabner M et al (2003) Elective cesaren section vs. spontaneous delivery: a comparative study of birth experience. Acta Obstet Gynecol Scand 82(9):834-840

4. Vogl SE, Worda C, Egarter C et al (2006) Mode of delivery is associated with maternal and fetal endocrine stress response. BJOG 113:441-445

\section{M. von Wolff , P. Stute}

\section{Gynäkologische Endokrinologie und Reproduktionsmedizin}

Das Praxisbuch

Stuttgart: Schattauer GmbH 2013,

1. Auflage, $476 \mathrm{~S}$., 42 Abb., $95 \mathrm{Tab}$. (ISBN 978-3-7945-2792-2), 89,99 EUR

Mit Michael von Wolff und Petra Stute legen zwei der besonders renommierten gynäkologischen Endokrinologen und Reproduktionsmediziner im deutsch-sprachigen Raum mit "Gynäkologische Endokrinologie und Reproduktionsmedizin - Das Praxisbuch" ein komplexes Handbuch für die Praxis vor, das auf 450 Seiten umfassend praktisch alle Aspekte der gynäkologischen Endokrinologie und Fortpflanzungsmedizin sowie viele spezielle Fragestellungen anspricht. Das Buch zielt in besonderem Maße auf die Verfügbarkeit eines schnellen Nachschlagewerks zu aktuellen klinischen Fragen, so ist es denn auch gegliedert.

20 Kapitel befassen sich mit überwiegend endokrinologischen Fragen, weitere 20 Kapitel mit allen Themen rund um Fortpflanzung und Reproduktionsmedizin. Dabei beschreiten die Autoren in der Klassifikation durchaus neue Wege und geben alt hergebrachte Einteilungsschemata auf. So findet der Leser in der gynäkologischen Endokrinologie sowohl praxisrelevante Darstellungen unter Oberbegriffen der verschiedenen Zyklusauffälligkeiten als auch altersbezogene Störungen, wie z.B. im Kapitel „Störungen der Geschlechtsentwicklung“ oder im Kapitel „Jugendendokrinologie oder Menopause und klimakterisches Syndrom".

Jedes Kapitel startet mit einem Absatz über für dieses Kapitel relevanten Definitionen, was die Arbeit deutlich erleichtert. Auch finden sich in vielen Kapiteln praxisrelevante Flußdiagramme, die einem die praktische $\mathrm{He}$ rangehensweise auch optisch nachvollziehbar darlegen. Den Schwerpunkten der Autoren entsprechend haben einige Kapitel auch in Ergänzung zu den üblichen Einteilungen eines solchen Lehrbuches besondere Berücksichtigung gefunden, wie z.B. die prämature Ovarialinsuffizienz, das natural cycle IVF oder die in vitro Maturation.

Dies ist auch gut, sucht man doch in den meisten Kompendien dieser Art vergeblich nach den sog.,Exoten“ des Faches.
Der Anspruch auf umfassende praxisrelevante Darstellungen ist hoch und natürlich auf 450 Seiten kaum in aller Tiefe darstellbar. Dies merkt man auch an einigen wenigen Kapiteln des Buches, in denen über allgemeine Therapieempfehlungen nicht hinausgegangen werden kann.

Insgesamt gesehen erlaubt das Buch aber für die Dichte der bearbeiteten Themen ein erstaunliches Maß auch an genauen praxisrelevanten Empfehlungen.

Der Leser wird besonders davon profitieren, dass er sich schnell einzelne unmittelbar aktuell für ihn wichtige kurze Themen herausgreifen kann und dann hier kompakt Antworten findet. So ist z.B. das Kapitel Endometriose kompetent gebündelt und die wirklich wichtigen Punkte, die für die Praxis und die Patientenberatung interessieren auf eben 5 Seiten zusammengefasst.

Insgesamt ein sehr gutes Werk für die schnelle Orientierung, das trotz seiner Komplexität und seinem Anspruch das Fach umfassend darzustellen, in einzelnen Absätzen erstaunlich detaillierte Informationen bietet. Der Preis von Euro 89,99 ist für ein Buch dieser Bedeutung fast konkurrenzlos.

T. Strowitzki (Heidelberg) 